\title{
SUBDIRECT SUMS OF INTEGERS AND REALS
}

\author{
PAUL F. CONRAD 1
}

1. Introduction and statement of the main theorems. The concept of a subdirect sum of integers is important in the study of latticeordered groups ("l-groups") since Weinberg [12] has shown that a free abelian $l$-group is a subdirect sum of integers and hence each abelian $l$-group is a homomorphic image of a subdirect sum of integers. In this paper those $l$-groups which are subdirect sums of integers are characterized. We also characterize those $l$-groups which are subdirect sums of subgroups of the naturally ordered additive group $R$ of real numbers. Topping [10] has shown that each vector lattice is a homomorphic image of such an $l$-group.

Pappert [9] has determined a necessary and sufficient condition for a vector lattice to be a subdirect sum of reals, and Bernau [2] has shown that with a slight modification her theory applies to an arbitrary $l$-group. Both of these authors use the fact that an archimedean $l$-group can be represented by almost finite functions on a Stone space to obtain their results. Our condition is simpler and the proof is elementary.

In [3] Bernau characterizes those subdirect sums of integers which contain the small sum and those which contain a dense subset of bounded elements. We can also characterize these classes of $l$-groups. These and other special cases and corollaries of our two main theorems are contained in $\$ 3$.

For each $\lambda \in \Lambda$ let $G_{\lambda}$ be a totally ordered group (" $o$-group") that is $o$-isomorphic to a subgroup of $R$. Thus each $G_{\lambda}$ is an archimedean $o$-group or equivalently an $o$-group without proper convex subgroups. $\pi G_{\lambda}$ will denote the large or unrestricted direct sum of the $G_{\lambda}$ ordered pointwise-the large cardinal sum of the $G_{\lambda}$-and $\sum G_{\lambda}$ will denote the small cardinal sum of the $G_{\lambda}$. In particular, $\pi G_{\lambda}$ is an $l$-group and $\sum G_{\lambda}$ is an $l$-ideal of $\pi G_{\lambda}$. If there exists an $l$-isomorphism of an $l$-group $G$ onto a subdirect sum of $\pi G_{\lambda}$, then we say that $G$ is a subdirect sum of reals. If, in addition, each $G_{\lambda}$ is cyclic, then we say that $G$ is a subdirect sum of integers.

Let $G$ be an $l$-group, $G^{+}=\{g \in G \mid g>0\}$ and let $Z^{+}$be the set of all strictly positive integers. An element $x \in G^{+}$will be called real if there exists a map $y \rightarrow \bar{y}$ of $G^{+}$into $Z^{+}$such that

Received by the editors May 4, 1967.

1 This research was supported by a grant from the National Science Foundation. 
I. $(\bar{y} x-y) \wedge(\bar{z} x-z) \neq 0$ for all $y, z \in G^{+}$. If, in addition, for all $y \in G^{+}$and all $n \in Z^{+}$,

II. $\bar{y}=1$ implies

$$
\overline{n y}=1,2
$$

III. $x \geqq 2 y$ implies $\bar{y}=1$,

then $x$ will be called an integral element of $G$.

THeOREM 1. An l-group $G$ is a subdirect sum of reals if and only if each $y \in G^{+}$exceeds a real element.

TheOREM 2. An l-group $G$ is a subdirect sum of integers if and only if each $y \in G^{+}$exceeds an integral element.

2. Proofs of Theorems 1 and 2. In all that follows, let $G \neq 0$ be an $l$-group. A convex $l$-subgroup $M$ of $G$ is a subgroup that satisfies

$$
|x| \leqq|a| \text { for } x \in G \text { and } a \in M \text { implies } x \in M \text {, }
$$

or, equivalently, $M$ is a sublattice and a convex subset of $G$. In particular, the set of all right cosets of a convex $l$-subgroup $M$ is a distributive lattice such that for all $a, b \in G$

$$
M+a \bigvee M+b=M+a \bigvee b
$$

and dually, where by definition $M+a \geqq M+b$ if $x+a \geqq b$ for some $x \in M$. A prime subgroup of $G$ is a convex $l$-subgroup for which the lattice of right cosets is totally ordered. For a convex $l$-subgroup $M$ of $G$ the following are equivalent.

(a) $M$ is prime.

(b) The set of convex $l$-subgroups that contain $M$ is a chain with respect to inclusion.

(c) If $a, b \in G^{+} \backslash M$, then $a \wedge b \in G^{+} \backslash M$.

Let $\mathscr{T}$ be the set of all maximal prime subgroups of $G$. If $M \in \mathscr{T}$ and $M \triangleleft G$, then $G / M$ is $o$-isomorphic to a subgroup of $R$ (notation $G / M \prec R)$. For proofs of the above see [6].

We shall consider the following properties of $x \in G^{+}$.

(1) There exists $M \in \mathscr{T}$ such that $M+x$ covers $M$ and for each $y \in G^{+}, M+n x>M+y$ for some $n \in Z^{+}$.

(2) $x$ is an integral element of $G$.

(3) $x$ is a real element of $G$.

(4) There exists $M \in \mathscr{N}$ such that for each $y \in G^{+}, M+n x>M+y$ for some $n \in Z^{+}$.

2 This element will be denoted by $(n y)^{-}$. 
Lemma. (1) $\rightarrow(2) \rightarrow(3) \leftrightarrow(4)$, and if each $M \in \mathfrak{T}$ is normal in $G$, then $(2) \rightarrow(1)$.

Proof. It follows from the definition of real and integral elements that $(2) \rightarrow(3)$. (4) $\rightarrow(3)$. For each $y \in G^{+}$let $\bar{y}$ be the least element in $Z^{+}$such that $M+\bar{y} x>M+y$. Then for all $y, z \in G^{+}$

$$
M+(\bar{y} x-y) \wedge(\bar{z} x-z)=M+(\bar{y} x-y) \wedge M+(\bar{z} x-z)>M .
$$

Thus $(\bar{y} x-y) \wedge(\bar{z} x-z) \neq 0$ and so $x$ is real.

$(1) \rightarrow(2)$. Define $\bar{y}$ as above. Since $M+x$ covers $M$, for $y \in G^{+}$and $n \in Z^{+}$the following are equivalent: $\bar{y}=1, y \in M, n y \in M$ and $(n y)^{-}$ $=1$. If $y \in G^{+}$and $x \geqq 2 y$, then $y \in M$ and so $\bar{y}=1$. For if $y \notin M$, then $M+x \geqq M+2 y>M+y>M$, but this contradicts the fact that $M+x$ covers $M$. Therefore $x$ is an integral element in $G$.

$(3) \rightarrow(4)$. For $y, z \in G^{+}$,

$[(\bar{y} x-y) \vee 0] \wedge[(\bar{z} x-z) \vee 0]=[(\bar{y} x-y) \wedge(\bar{z} x-z)] \vee 0 \in G^{+}$.

Thus $Q_{x}=\left\{(\bar{y} x-y) \vee 0 \mid y \in G^{+}\right\}$is contained in an ultrafilter $K$ of $G^{+}$. That is, $0<a \wedge b \in K$ for all $a, b \in K$, and $K$ is maximal with respect to this property. It follows that $N=\mathrm{U}_{k \in K} k^{\prime}$ is a minimal prime subgroup of $G$ and $K=G^{+} \backslash N$, where $k^{\prime}=\{g \in G|| g \mid \wedge k=0\}$ is the polar of $k$. This is Theorem 5.1 in [7], and this result is also implicit in [1] and $[8]$.

(A) $N+\bar{y} x>N+y$ for each $y \in G^{+}$. For we have $(\bar{y} x-y) \vee 0 \in K$ $=G^{+} \backslash N$ and hence $N+(\bar{y} x-y) \bigvee 0>N$ and so $N+\bar{y} x-y>N$. Since the convex $l$-subgroups of $G$ that contain $N$ form a chain, there is a unique convex $l$-subgroup $M \supseteq N$ that is maximal with respect to $x \notin M$.

(B) $M \in \mathscr{M}$. For if $y \in G^{+}$, then $N+\bar{y} x>N+y$ and hence $a+\bar{y} x>y>0$ for some $a \in N$. But clearly $a+\bar{y} x$ is contained in any convex $l$-subgroup that properly contains $M$. Therefore $G$ covers $M$ and hence $M \in \mathscr{T}$. It follows from (A) that

$$
M+(\bar{y}+1) x>M+\bar{y} x \geqq M+y .
$$

Therefore (4) is satisfied.

To complete the proof we need to show that $(2) \rightarrow(1)$ provided that each $M \in \mathscr{N}$ is normal in $G$. Let $x$ be an integral element and let $M$ and $N$ be as above. Suppose (by way of contradiction) that $M+x>M+y>M$ for some $y \in G$. Then since $M+y \bigvee 0=M+y \bigvee M$ $=M+y$ and $M+x \wedge y=M+x \wedge M+y=M+y$, we may assume that $x>y>0$. Now $x=x-y+y$ and since $x-y, y \in G^{+} \backslash M$ and $M$ is prime, $d=(x-y) \wedge y \in G^{+} \backslash M$. Clearly $x \geqq 2 d$ and hence $\bar{d}=1$ and $(n d)^{-}=1$ 
for all $n \in Z^{+}$. Thus $M+x=M+(n d)^{-} x \geqq M+n d \geqq M+d>M$ for all $n \in Z^{+}$, but this is impossible because $G / M \prec R$.

REMARK. S. Wolfenstein and T. Lloyd have independently shown that if $M$ is a prime subgroup and $M+x$ covers $M$, then $M$ is normal in the convex $l$-subgroup that covers it. Thus (1) is equivalent to

$\left(1^{\prime}\right)$ There exists $M \in \mathscr{T}$ such that $M+x$ covers $M$.

Corollary. Suppose that each $M \in \mathfrak{N}$ is normal in $G$ and consider $x \in G^{+}$.

(a) $x$ is a real element of $G$ if and only if $x \in G \backslash M$ for some $M \in \mathscr{T}$.

(b) $x$ is an integral element of $G$ if and only if $M+x$ covers $M$ for some $M \in \mathscr{T}$.

Proof. This is an immediate consequence of the lemma and the fact that $G / M \prec R$ is an archimedean $o$-group for each $M \in \mathscr{N}$.

Byrd [4] has shown that $G$ is a subdirect sum of 0 -groups if and only if for each prime subgroup $M$ and each $g \in G,-g+M+g \subseteq M$ or $-g+M+g \supseteq M$. Thus for this class of $l$-groups each $M \in \mathscr{T}$ is normal.

Proof of Theorem 1. Suppose that $G$ is a sublattice and a subdirect sum of $\pi R_{\lambda}(\lambda \in \Lambda)$, where each $R_{\lambda} \subseteq R$. If $x \in G^{+}$, then $x_{\lambda}>0$ for some $\lambda \in \Lambda$. Let $M=\left\{g \in G \mid g_{\lambda}=0\right\}$. Then $M \in \mathscr{T}$ and $x \in G \backslash M$. Thus by the corollary, $x$ is real, and so each $x \in G^{+}$is real.

Conversely suppose that each element in $G^{+}$exceeds a real element, and consider $y, z \in G^{+}$. There exists a real element $x \leqq z$. Thus $\bar{y} x \leq y$ and hence $\bar{y} z y$. Therefore $G$ is archimedean and hence abelian. By the corollary, $x \in G \backslash M$ for some $M \in \mathscr{T}$ and hence $z \in G \backslash M$. Therefore $0=\bigcap\{M \mid M \in \Re\}$ and so $G$ is a subdirect sum of reals.

Proof of Theorem 2. Suppose that $G$ is a sublattice and a subdirect sum of $\pi Z_{\lambda}(\lambda \in \Lambda)$, where each $Z_{\lambda}=Z$. If $g \in G^{+}$, then $g \geqq x>0$ for some $x \in G$ where $x_{\lambda}=1$ for some $\lambda \in \Lambda$. Let $M=\left\{g \in G \mid g_{\lambda}=0\right\}$. Then $M \in \mathscr{M}$ and $M+x$ covers $M$, and hence by the corollary $x$ is integral. Therefore each element in $G^{+}$exceeds an integral element.

Conversely, suppose that each element in $G^{+}$exceeds an integral element. Then, as in the proof of Theorem $1, G$ is abelian. Let $g$ $=\{M \in \mathscr{T} \mid G / M$ is cyclic $\}$. Then by the corollary $\cap\{M \mid M \in g\}$ must be zero since it contains no integral element. Therefore $G$ is a subdirect sum of in tegers.

3. Special cases of Theorems 1 and 2. An element $s \in G^{+}$is called basic if $\{g \in G \mid 0 \leqq g \leqq s\}$ is totally ordered.

Proposition A. For an l-group $G$ the following are equivalent.

(1) $G$ is a subdirect sum of reals that contains the small sum. 
(2) Each element in $G^{+}$exceeds a real element that is also basic.

(3) $G$ is archimedean and each element in $G^{+}$exceeds a basic element.

Proof. It is shown in [5] that (1) $\leftrightarrow(3)$. If each element in $G^{+}$ exceeds a real element, then $G$ is archimedean and hence $(2) \rightarrow(3)$. If (1) holds, then each element in $G^{+}$is real, and hence (1) and (3) imply (2).

There are many other equivalent conditions proven in the literature-see for example [11].

An element $a \in G^{+}$is an atom if it covers 0 . It is shown in [5] that $x$ is a basic element in an archimedean $l$-group $G$ if and only if $x^{\prime \prime} \prec R$ and $G$ is the cardinal sum of $x^{\prime \prime}$ and $x^{\prime}$. Thus a basic element $x$ is integral if and only if $x^{\prime \prime}$ is cyclic, and hence if and only if $x$ is an atom.

Proposition B. For an l-group $G$ the following are equivalent.

(1) $G$ is a subdirect sum of integers that contains the small sum.

(2) Each element in $G^{+}$exceeds an integral element that is also basic.

(3) $G$ is archimedean and each element in $G^{+}$exceeds an atom.

Proof. Clearly (1) $\rightarrow(2) \rightarrow(3)$.

$(3) \rightarrow(1)$. Since each atom is a basic element it follows from Proposition A that $G$ is a subdirect sum of reals that contains the small sum. Thus without loss of generality

$$
\sum R_{\lambda} \subseteq G \subseteq \pi R_{\lambda},
$$

where $R_{\lambda} \subseteq R$ for each $\lambda \in \Lambda$. If $R_{\lambda}$ is not cyclic, then there exists an element in $R_{\lambda}^{+} \subseteq G^{+}$that does not exceed an atom, a contradiction. Therefore (1) holds.

An element $s \in G^{+}$is called singular if $a \wedge(s-a)=0$ for each $0 \leqq a \leqq s$.

Proposition C. For an l-group $G$ the following are equivalent.

(1) $G$ is a subdirect sum of integers and each element in $G^{+}$exceeds a bounded element.

(2) Each element in $G^{+}$exceeds an integral element that is also singular.

(3) $G$ is a subdirect sum of reals and each element in $G^{+}$exceeds a singular element.

Proof. In [7] it is shown that (1)↔(3) and clearly (2) $\rightarrow$ (3). Suppose that (1) and (3) hold. Then without loss of generality $G \subseteq \pi Z_{\lambda}$, where for each $\lambda \in \Lambda, Z_{\lambda}=Z$. In [7] it is shown that if $s \in G$ is singular, then $s_{\lambda}=1$ or 0 . Thus it follows that $s$ is integral and hence we have (2). 
Bernau [3] has established (1) $\leftrightarrow(3)$ in Proposition B and has derived a condition that is equivalent to (1) in Proposition $\mathrm{C}$.

Suppose that $x \in G^{+}$is real and let $A_{x}$ be the set of all maps $\pi: G^{+} \rightarrow Z^{+}$ such that for all $y, z \in G^{+}$

$$
((y \pi) x-y) \wedge((z \pi) x-z) \leq 0 .
$$

For $\alpha, \beta \in A_{x}$, define $\alpha \leqq \beta$ if $y \alpha \leqq y \beta$ for all $y \in G^{+}$. Then $\left(A_{x}, \leqq\right)$ is a po-set and each element in $A_{x}$ exceeds a minimal element in $A_{x}$. For if $\left\{\alpha_{\lambda} \mid \lambda \in \Lambda\right\}$ is a chain in $A_{x}$, then for each $y \in G^{+}$define

$$
y \pi=\min \left\{y \alpha_{\lambda} \mid \lambda \in \Lambda\right\} .
$$

If $y, z \in G^{+}$, then there exists $\lambda \in \Lambda$ such that $y \alpha_{\lambda}$ and $z \alpha_{\lambda}$ are minimal and so

$$
((y \pi) x-y) \wedge((z \pi)-z)=\left(\left(y \alpha_{\lambda}\right) x-y\right) \wedge\left(\left(z \alpha_{\lambda}\right) x-z\right) \neq 0 .
$$

Therefore $\pi \in A_{x}$, and hence by Zorn's lemma each map in $A_{x}$ exceeds a minimal map.

Definition. A real element $x \in G^{+}$for which there exists a minimal map $y \rightarrow \bar{y}$ in $A_{x}$ that also satisfies (II) will be called a *element.

Proposition D. For an l-group the following are equivalent.

(1) Each element in $G^{+}$exceeds $a^{*}$-element.

(2) $G$ is (l-isomorphic to) a subdirect sum of $\pi Z_{\lambda}$, where for each $\lambda \in \Lambda, Z_{\lambda}=Z$; and $G_{\lambda}=\left\{g \in G \mid g_{\lambda}=0\right\}$ is both a maximal and a minimal prime subgroup of $G$.

Proof. (1) $\rightarrow(2)$. Since each *-element is real, it follows from Theorem 1 that $G$ is abelian. Let $x$ be a *element in $G$ and let $y \rightarrow \bar{y}$ be a minimal map in $A_{x}$ that also satisfies (II). Construct $M$ and $N$ as in the proof of $(3) \rightarrow(4)$ in the lemma. Since $N+\bar{y} x>N+y$ for all $y \in G^{+}$and the map $y \rightarrow \bar{y}$ is minimal, it follows that $\bar{y}$ is the least element in $Z^{+}$for which $N+\bar{y} x>N+y$. Suppose (by way of contradiction) that $M \supset N$ and pick $0<z \in M \backslash N$ and let $y=-(x \wedge z)+x$. Then

$$
M+x=M+y \text { and } N+x>N+y .
$$

Therefore $\bar{y}=1$ and hence $(2 y)^{-}=1$, but clearly $N+(2 y)^{-} x=N+x$ $<N+2 y$, a contradiction. Thus $N=M$ is both maximal and minimal. If $M+x>M+y$, then $\bar{y}=1$ and hence $M+x=M+(n y)^{-} x \geqq M+n y$ for all $n \in Z^{+}$. Thus since $G / M \prec R$ it follows that $y \in M$ and so $G / M$ is cyclic.

$(2) \rightarrow(1)$. We may assume that $G \subseteq \pi Z_{\lambda}$. If $z \in G^{+}$, then $z \geqq x \in G^{+}$, where $x_{\lambda}=1$ for some $\lambda \in \Lambda$. For $y \in G^{+}$define $\bar{y}$ to be the least element 
in $Z^{+}$such that $\bar{y} x_{\lambda}>y_{\lambda}$. Then the map $y \rightarrow \bar{y}$ satisfies (I), (II) and (III). It remains to be shown that this map is minimal in $A_{x}$. Suppose that $\pi \in A_{x}$ and that $y \pi \leqq \bar{y}$ for all $y \in G^{+}$. Construct $M$ and $N$ as above using the map $y \rightarrow y \pi$. In particular, $N+(y \pi) x>N+y$ and $M+(y \pi) x$ $\geqq M+y$ for all $y \in G^{+}$.

If $M \neq G_{\lambda}$, then there exists $y \in G^{+}$such that $y_{\lambda}=0$ and $y \notin M$. Since $y_{\lambda}=0, \bar{y}=1$ and so $(n y)^{-}=(n y) \pi=1$ for all $n \in Z^{+}$, but this means that $M+x=M+((n y) \pi) x \geqq M+n y$ for all $n \in Z^{+}$, and this contradicts the fact that $G / M \prec R$.

If $M=G_{\lambda}$, then since $G_{\lambda}$ is a minimal prime $M=N$ and so $M+(y \pi) x$ $>M+y$ for all $y \in G^{+}$, and it follows that $\bar{y}=y \pi$ for all $y \in G^{+}$. Therefore $x$ is a *-element and so (1) is satisfied.

\section{REFERENCES}

1. B. Banaschewski, On lattice ordered groups, Fund. Math 55 (1964), 113-123.

2. S. Bernau, Unique representations of archimedean lattice groups and normal archimedean lattice rings, Proc. London Math. Soc. 15 (1965), 599-631.

3. - On subdirect sums of integers (preprint).

4. R. Byrd, Complete distributivity in lattice ordered groups, Pacific J. Math. 20 (1967), 423-432.

5. P. Conrad, Some structure theorems for lattice-ordered groups, Trans. Amer. Math. Soc. 99 (1961), 1-29.

6. - - The lattice of all convex l-subgroups of a lattice-ordered group, Czechoslovak Math. J. 15 (1965), 101-123.

7. P. Conrad and D. McAlister, The completion of a lattice ordered group, J. Austral. Math. Soc. (to appear).

8. D. Johnson and J. Kist, Prime ideals in vector lattices, Canad. J. Math. 14 (1962), 517-528.

9. D. Papert, A representation theory for lattice groups, Proc. London. Math. Soc. 12 (1962), 100-120.

10. D. Topping, Some homological pathology in vector lattices, Canad. J. Math. 17 (1965), 411-428.

11. E. Weinberg, Completely distributive lattice-ordered groups, Pacific J. Math. 12 (1962), 1131-1137.

12. - Free lattice ordered abelian groups, Math. Ann. 159 (1965), 217-222.

TULANE UNIVERSITY 